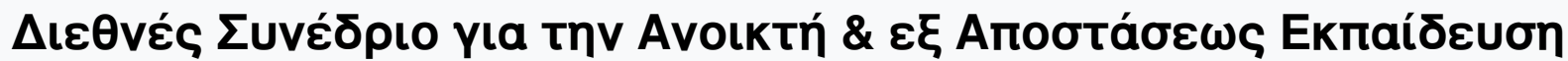

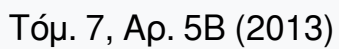

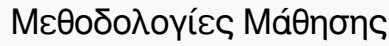

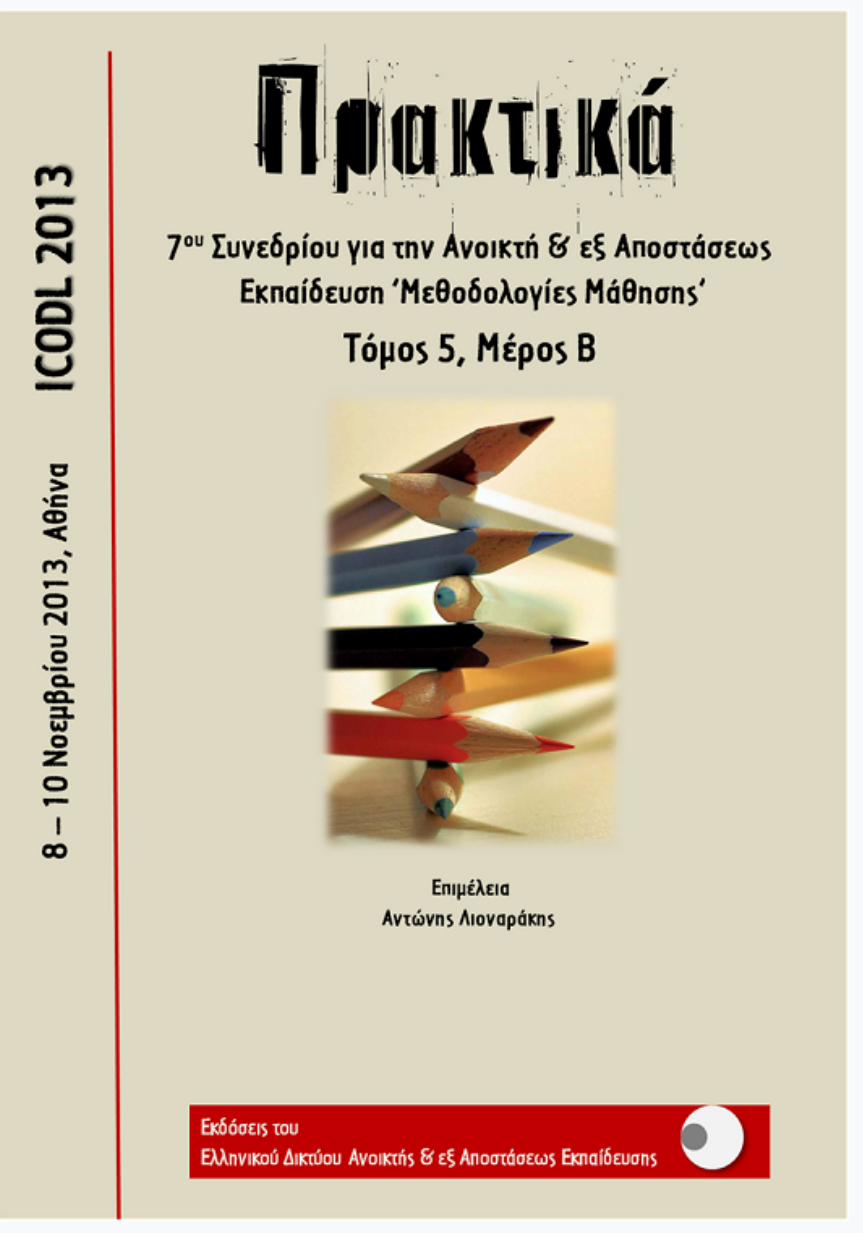

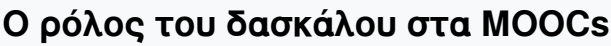

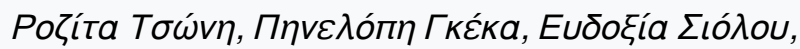

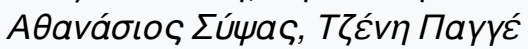

doi: $\underline{10.12681 / \text { icodl.561 }}$ 


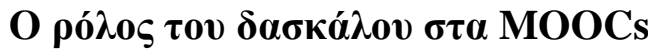

\section{The role of the tutor in MOOC}

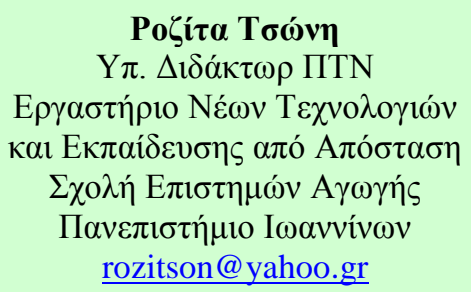

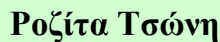

Y $\pi . \Delta \mathrm{t} \delta \alpha ́ \kappa \tau \omega \rho \Pi T N$

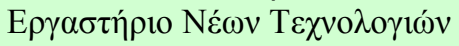

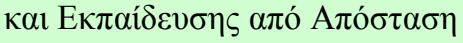

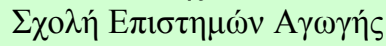

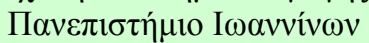
rozitson@yahoo.gr

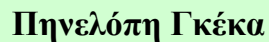 \\ Y $\pi . \Delta \mathrm{t} \delta \alpha ́ \kappa \tau \omega \rho ~ \Pi T N$

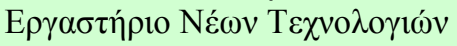

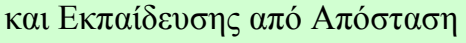

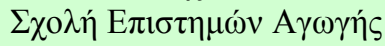

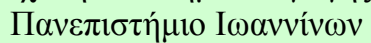 \\ paula@cc.uoi.gr
}

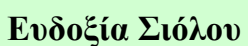

Y $\pi . \Delta \delta \delta \alpha ́ \kappa \tau \omega \rho \Pi T N$

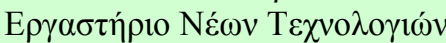

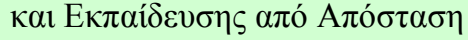

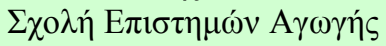

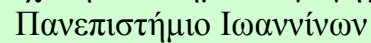
esiolou@yahoo.gr

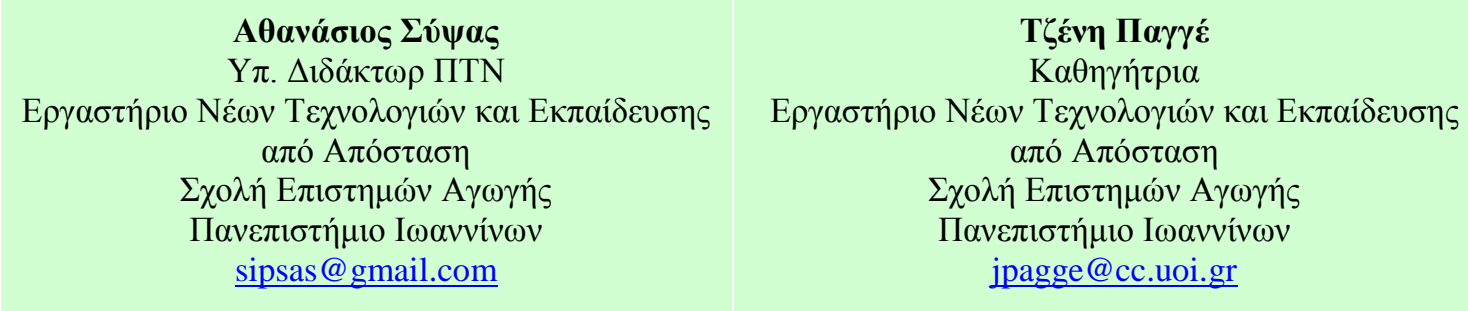

\begin{abstract}
In the transition from the traditional face to face learning to open online teaching, the teacher plays an important role in the process of learning. Educational models have given different characteristics to the teacher but all converge on a general consensus within the competencies required for successful educational tasks. The MOOCs (massive open online courses) are using the principles of Connectivism. So, in many cases the role of the teacher is downgraded while the learning process takes place independently. In these open classes, where participants are numerous, there is a debate about the involvement of the teacher with the students. Teaching assistants (TAs) are also very important to conduct the course. The aim of this review is to examine the role of the teacher during an online course as initiator and evaluator of the learning process. Moreover we discuss how open courses could be used, in other ways, and especially in universities for LLL.
\end{abstract}

Keywords: distance learning, open learning, MOOC, conectivism, tutor's role

\section{Пєрі́ $\eta \psi \eta$}

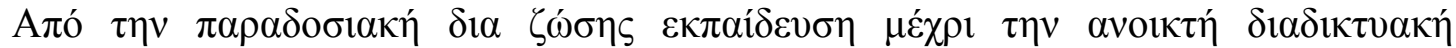

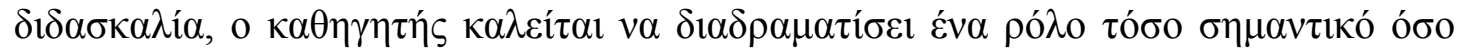

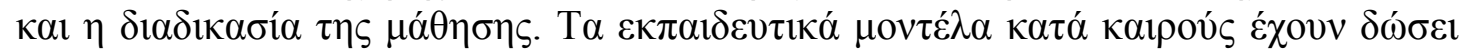

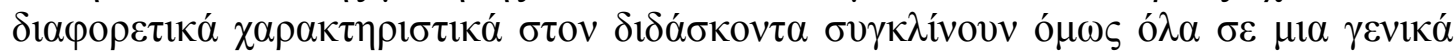

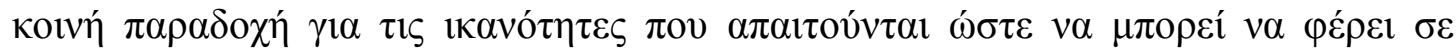

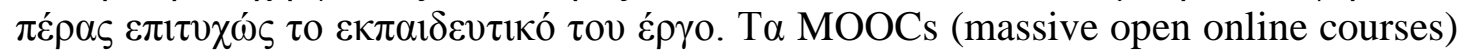

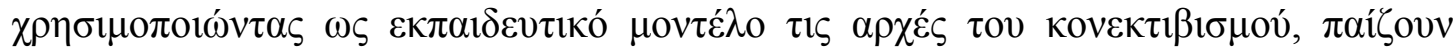

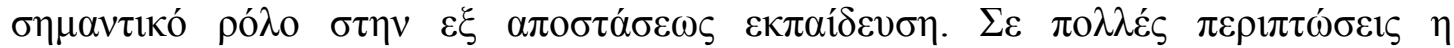

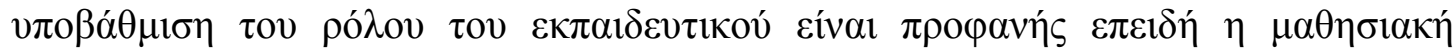

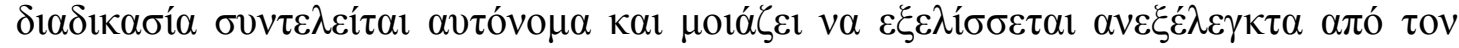




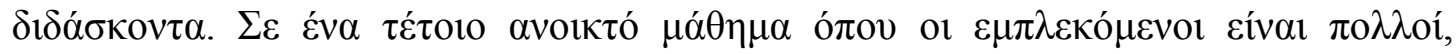

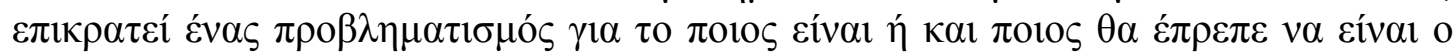

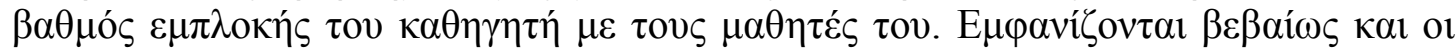

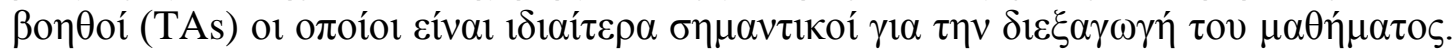

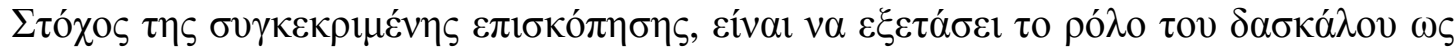

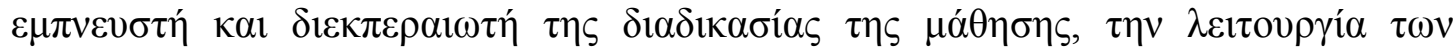

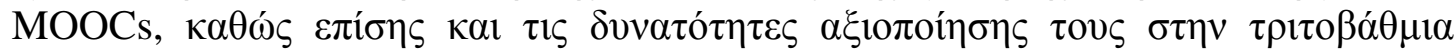
$\varepsilon \kappa \pi \alpha i ́ \delta \varepsilon v \sigma \eta$.

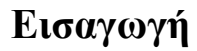

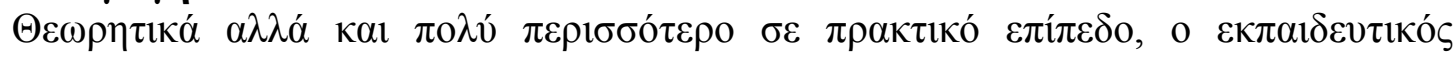

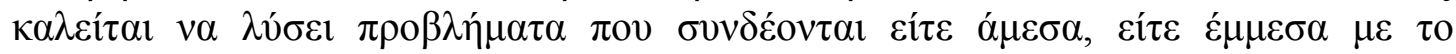

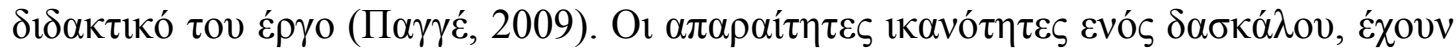

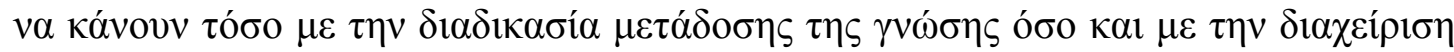

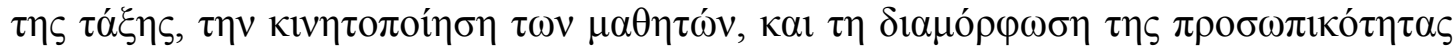

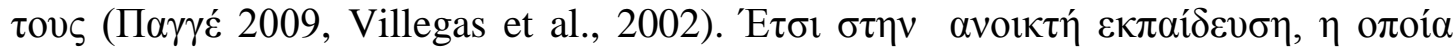

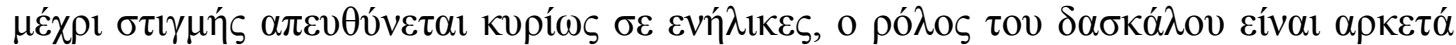

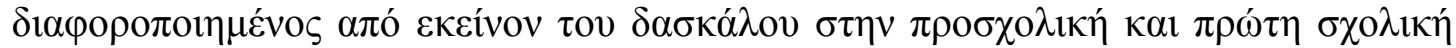

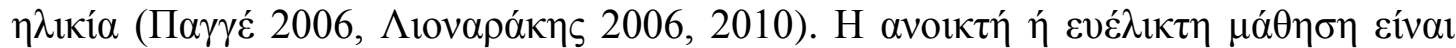

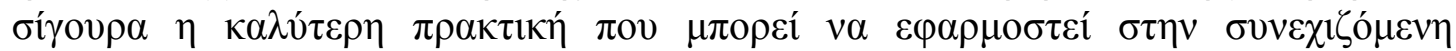
$\varepsilon \kappa \pi \alpha i ́ \delta \varepsilon v \sigma \eta \eta \dot{~ \sigma \tau \eta v ~ \varepsilon \kappa \pi \alpha i ́ \delta \varepsilon v \sigma \eta ~ \varepsilon v \eta \lambda i ́ \kappa \omega v ~(\Lambda i o v \alpha \rho \alpha ́ \kappa \eta \varsigma, ~ 2001, ~ П \alpha \gamma \gamma \varepsilon ́, ~ 2009, ~ M o o r e ~ e t ~}$

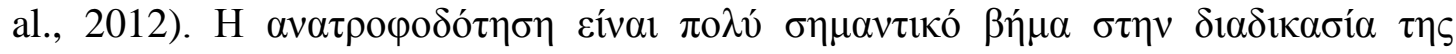

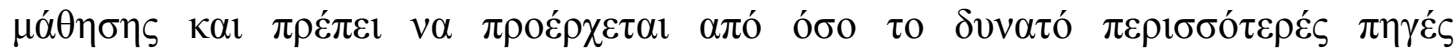

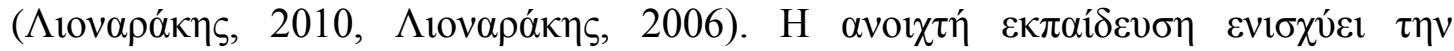

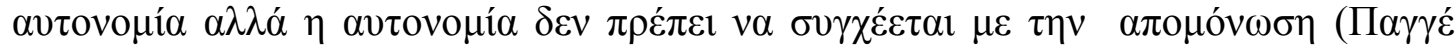

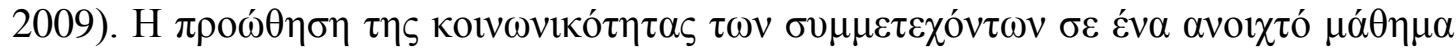

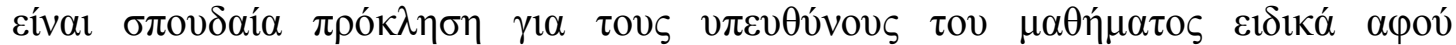

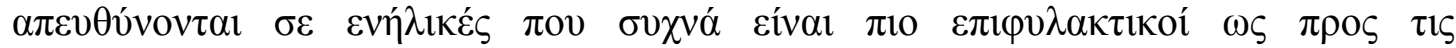

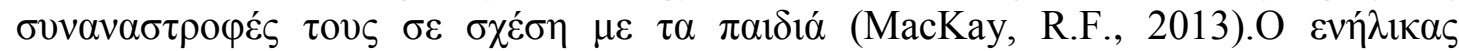

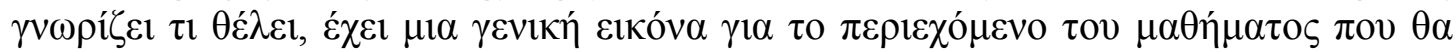

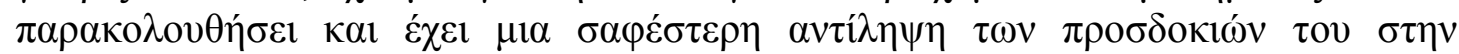

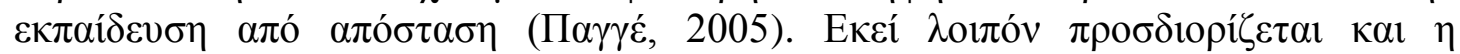

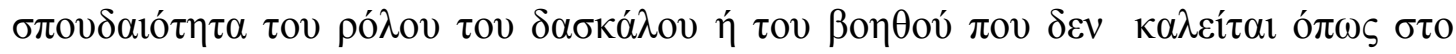

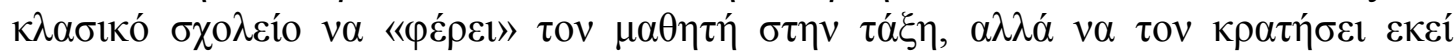

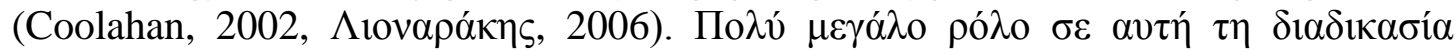

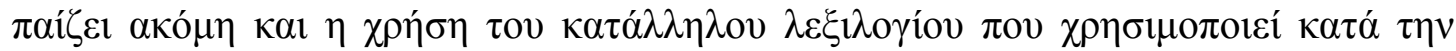



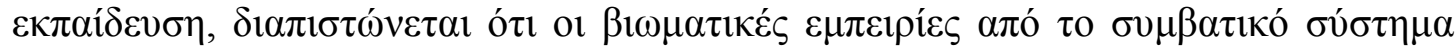

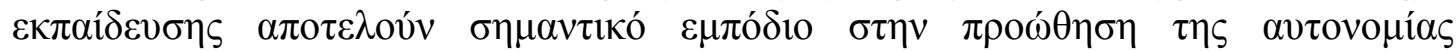

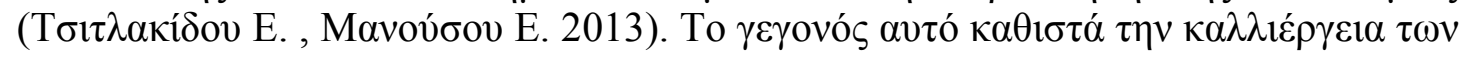

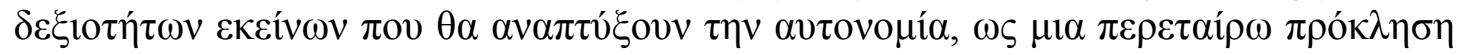

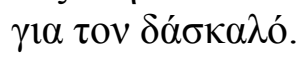

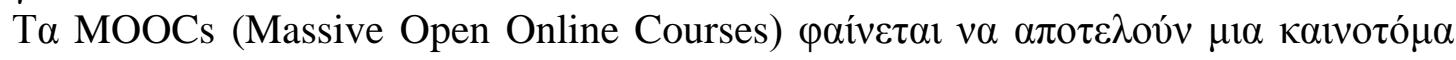

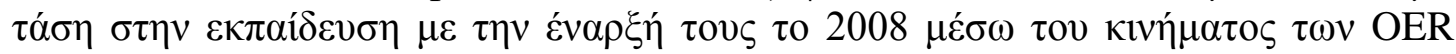

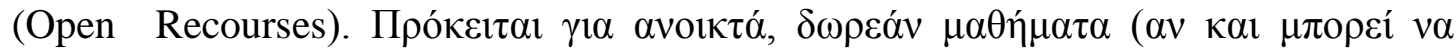

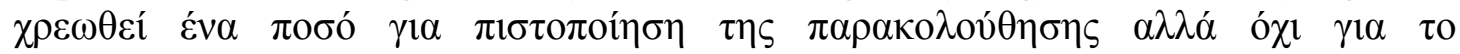

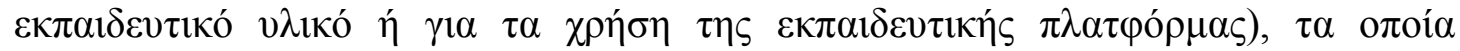

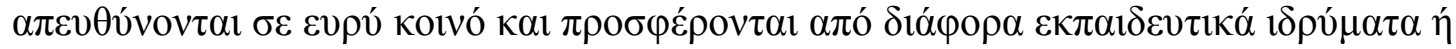

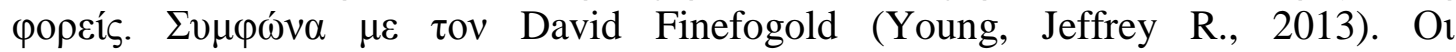




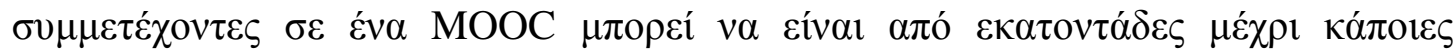

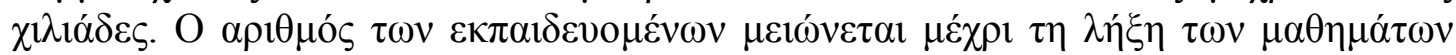

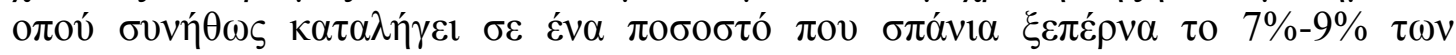

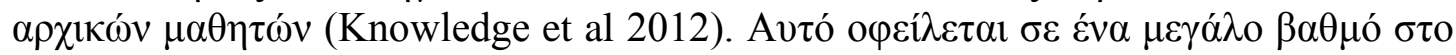

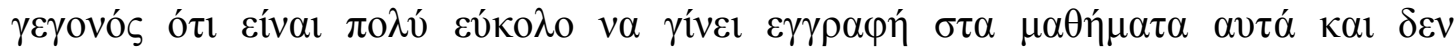

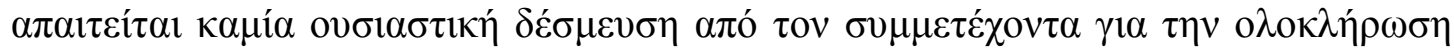

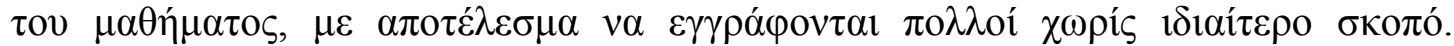



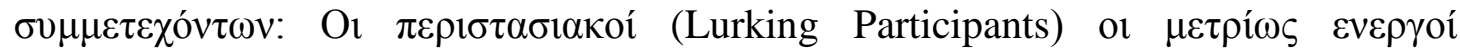

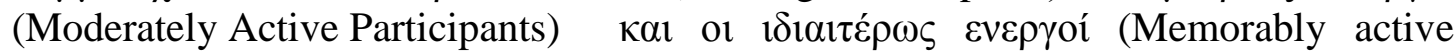

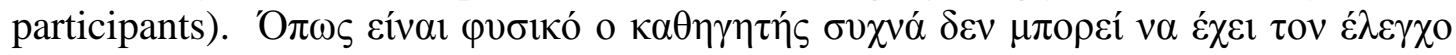

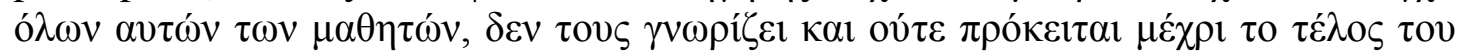

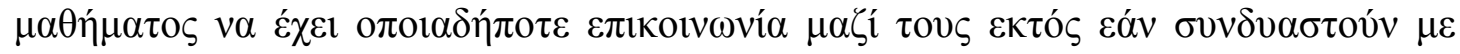

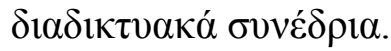

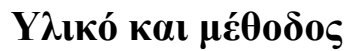

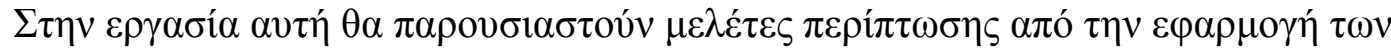

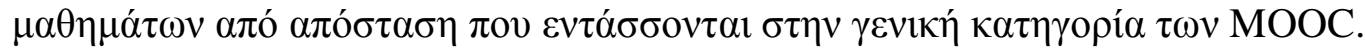

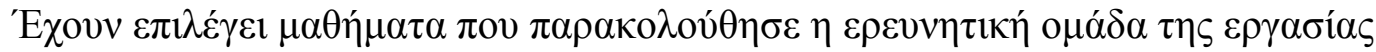

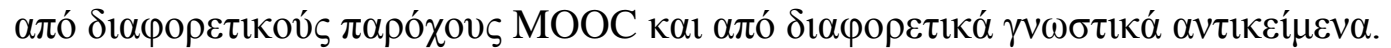

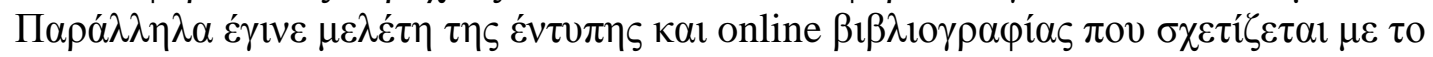

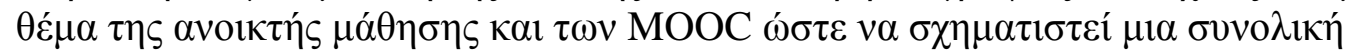

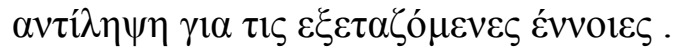

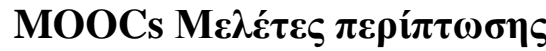

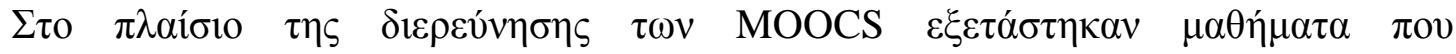

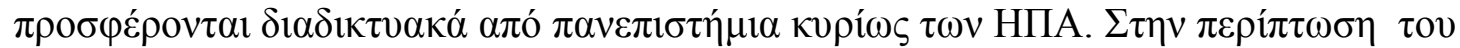

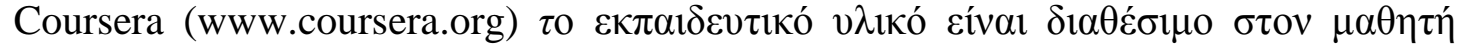

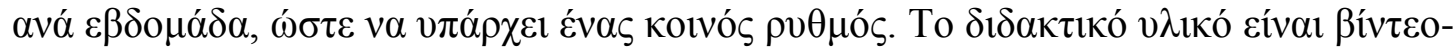

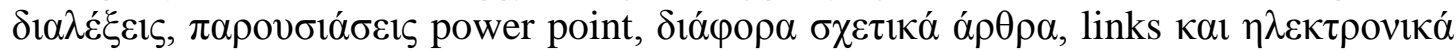

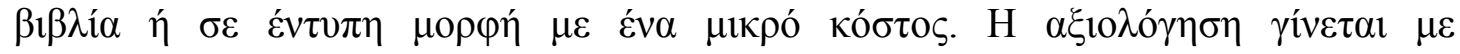

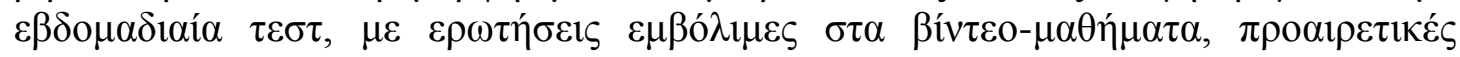

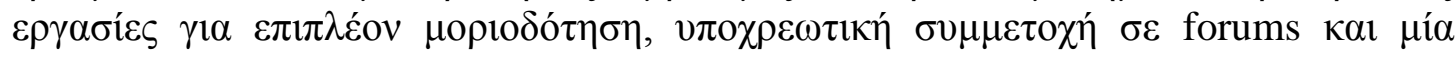

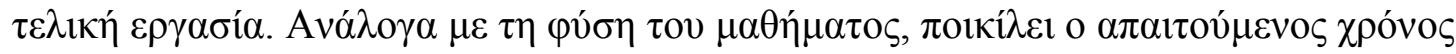

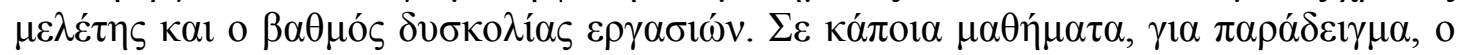

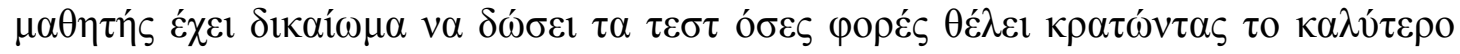

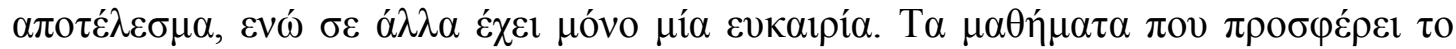

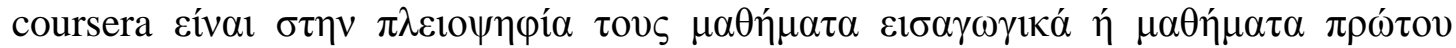

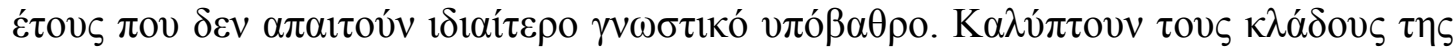

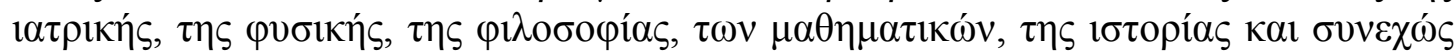

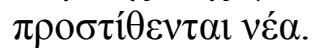

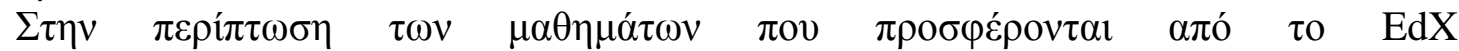

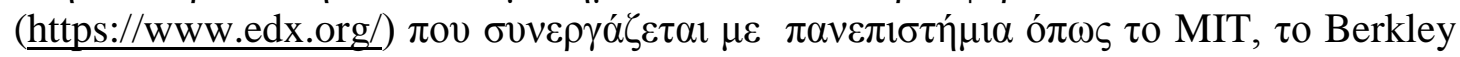

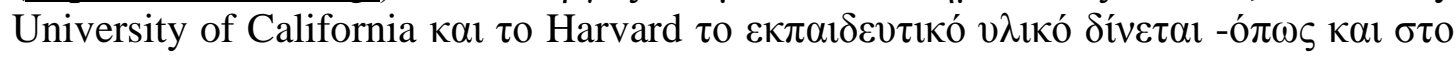

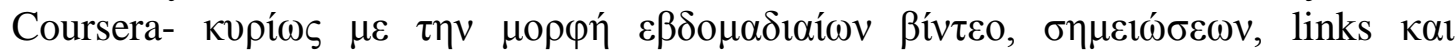

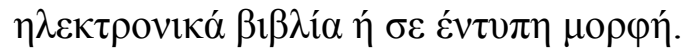

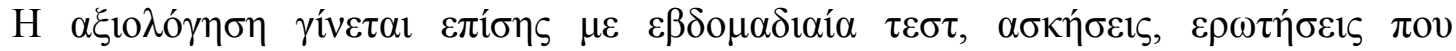

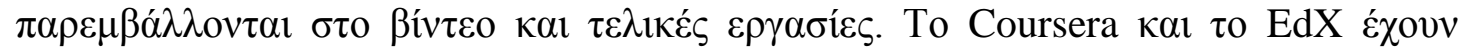

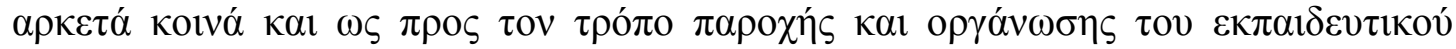

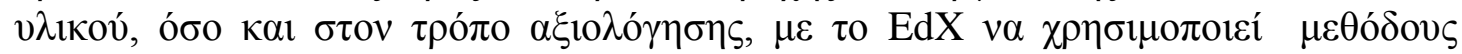




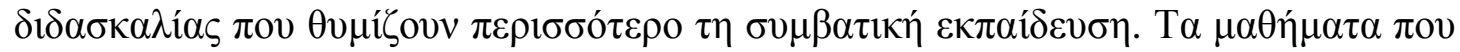

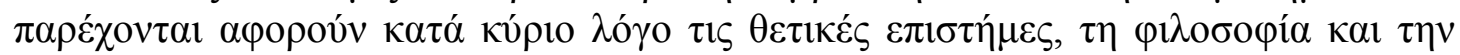
$1 \sigma \tau$ opía.

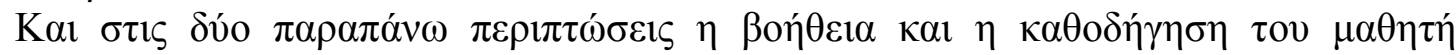

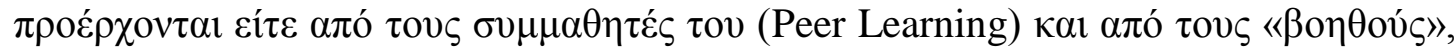

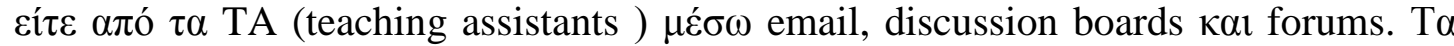

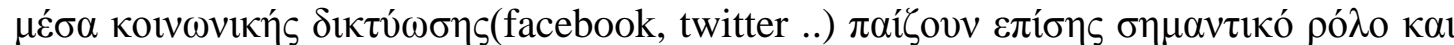

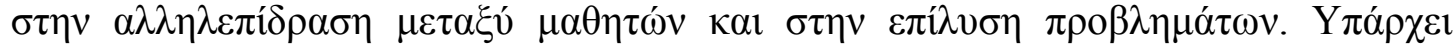

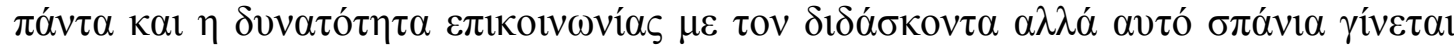

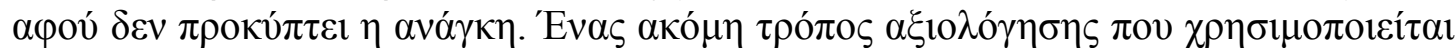

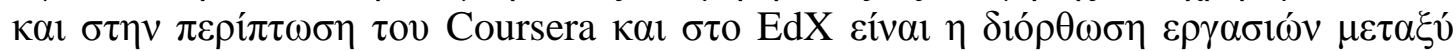

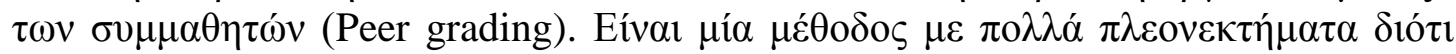

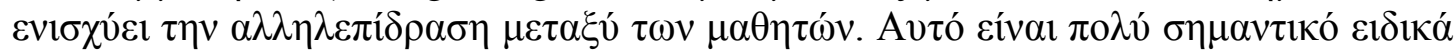

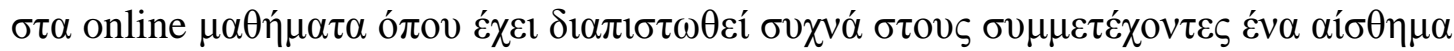

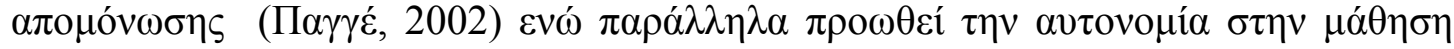

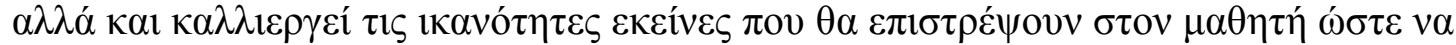

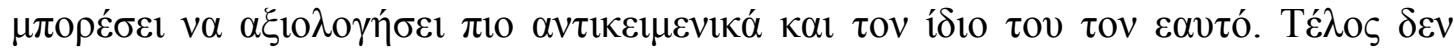

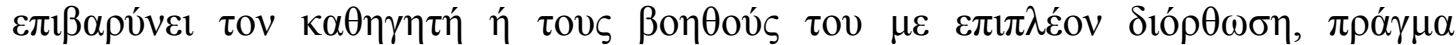

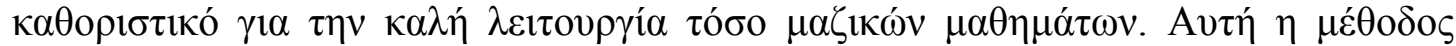

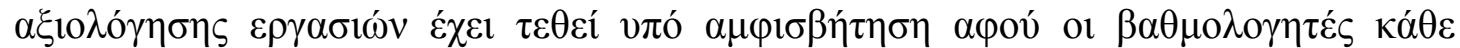

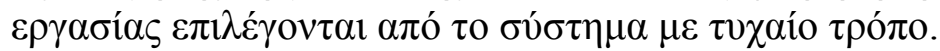

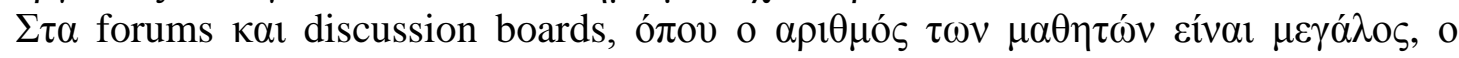

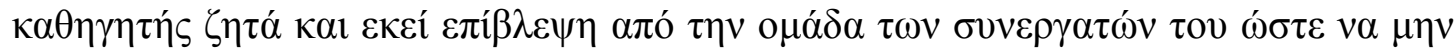

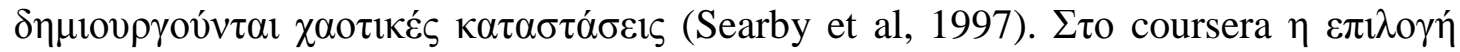

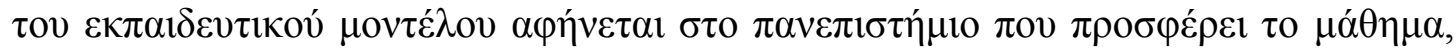

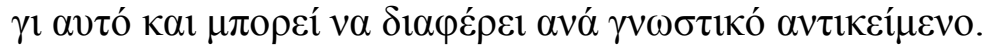

$\Sigma \tau \eta \nu \pi \varepsilon \rho i ́ \pi \tau \omega \sigma \eta \tau$

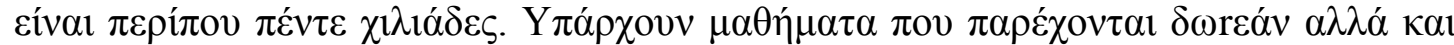

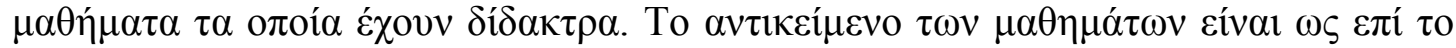

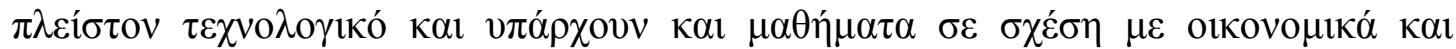

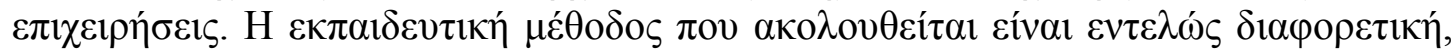

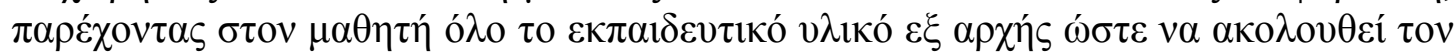

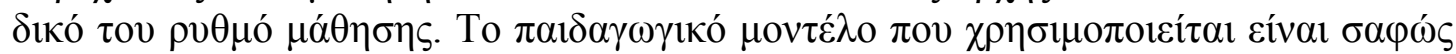

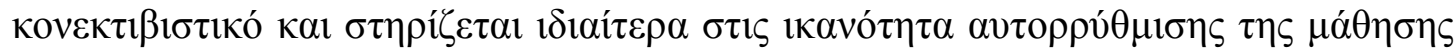

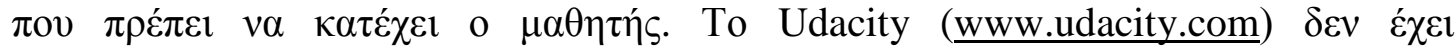

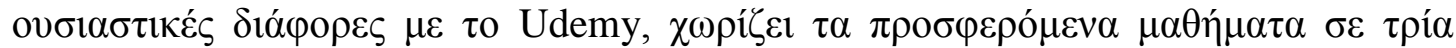



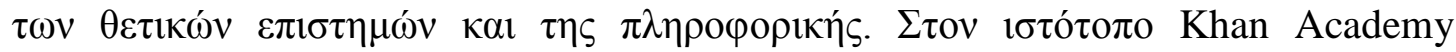

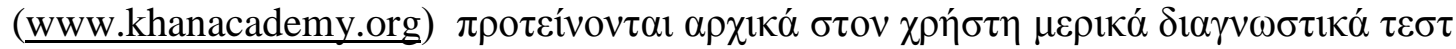

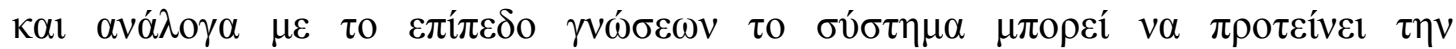

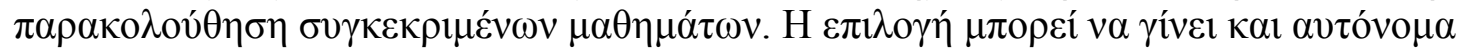
$\alpha \pi$ ó $\tau$ ov $\varepsilon \kappa \pi \alpha 1 \delta \varepsilon v o ́ \mu \varepsilon v o$.

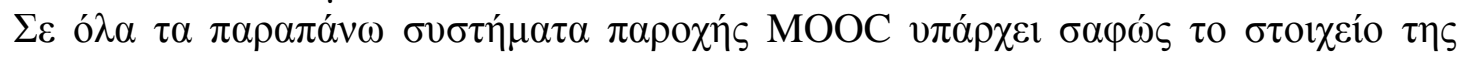

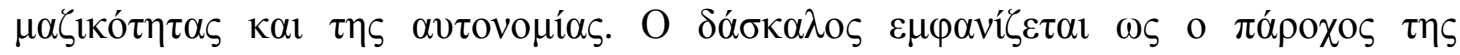

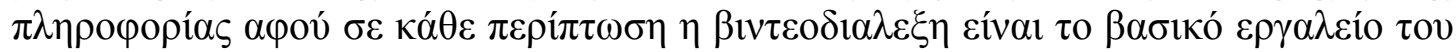

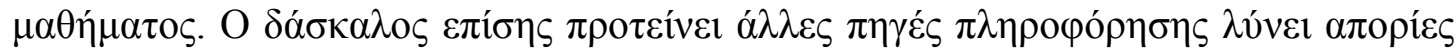

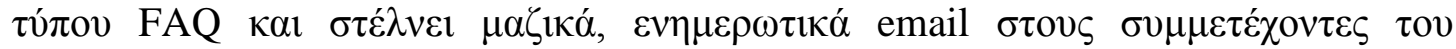

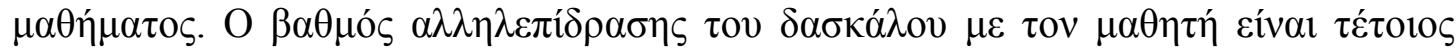

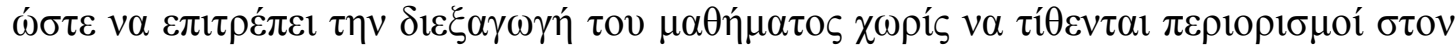

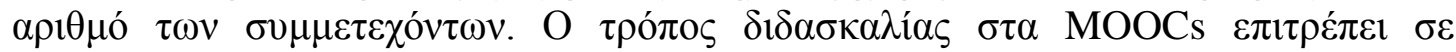




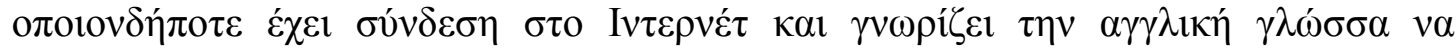

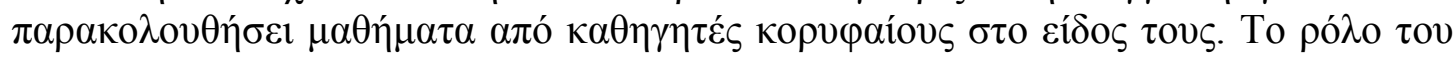

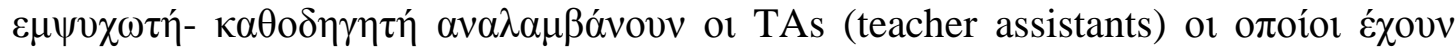

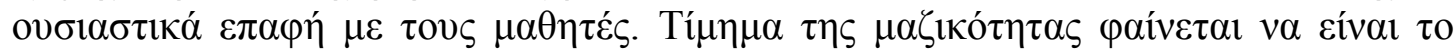

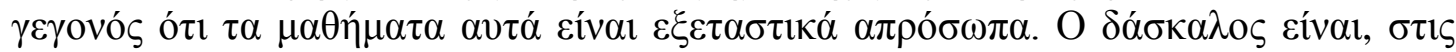

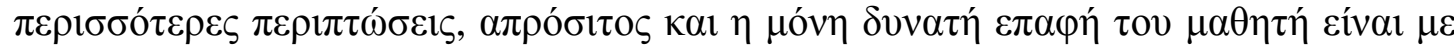

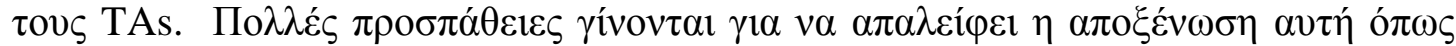

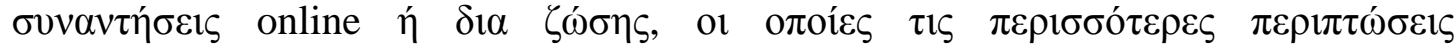

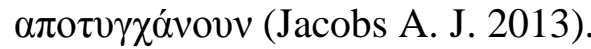

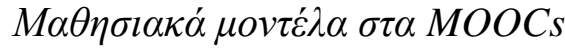

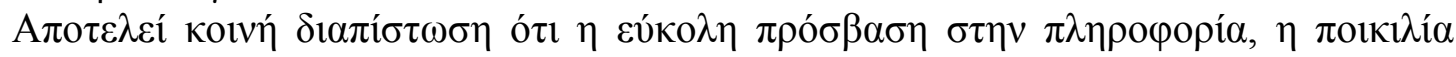

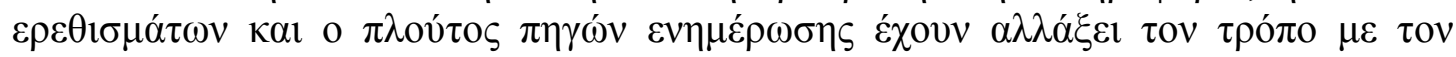

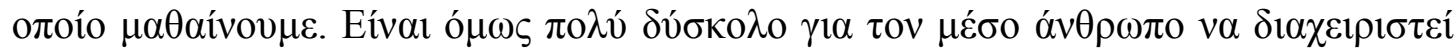

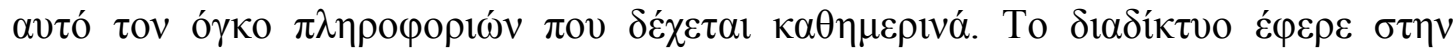

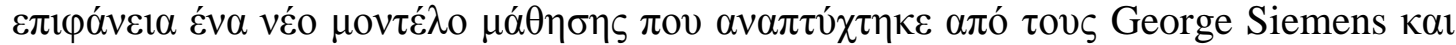

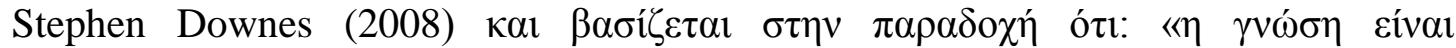

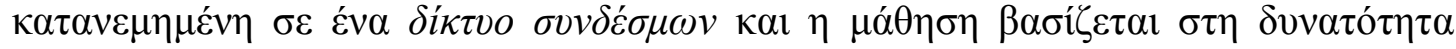

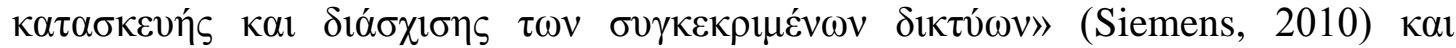

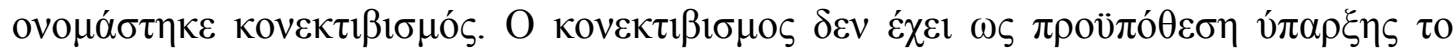

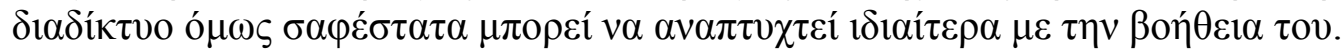

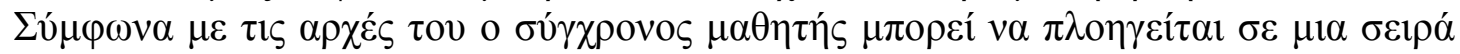

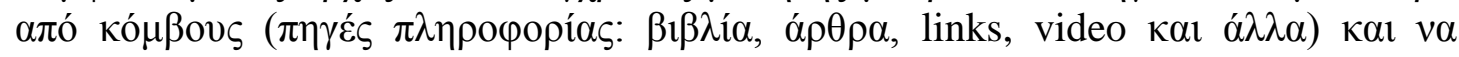

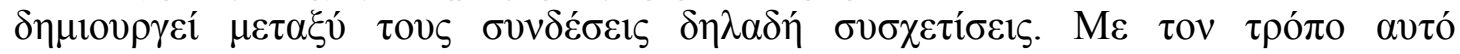

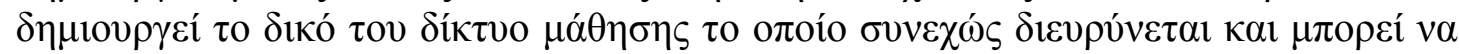

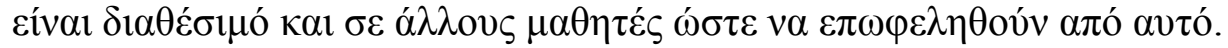

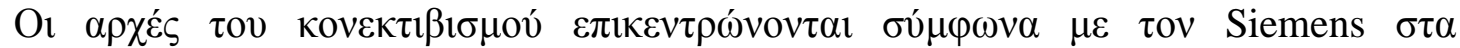

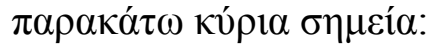

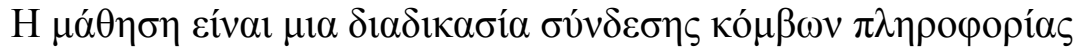

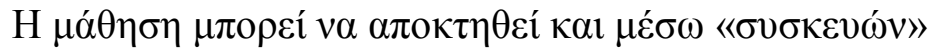

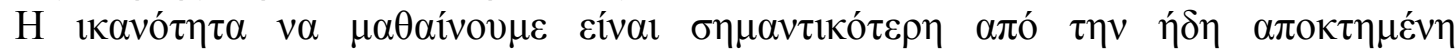
$\pi \lambda \eta \rho о \varphi о \rho i ́ \alpha ~(\gamma \nu \omega ́ \sigma \eta)$





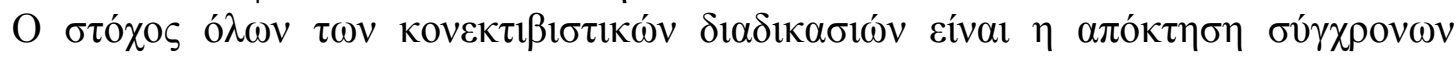

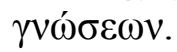

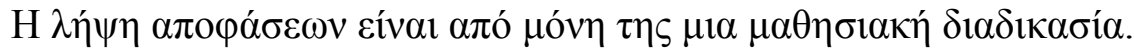

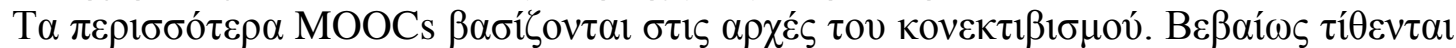

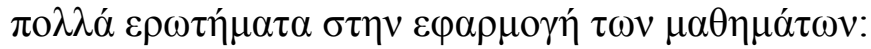

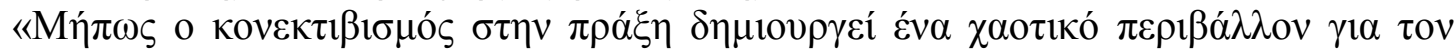

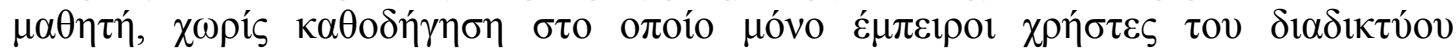

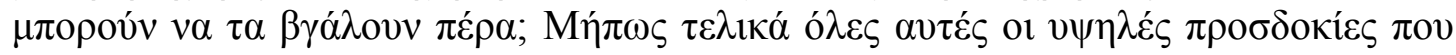

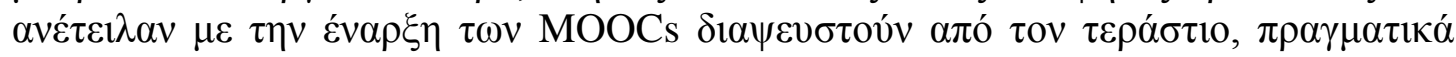

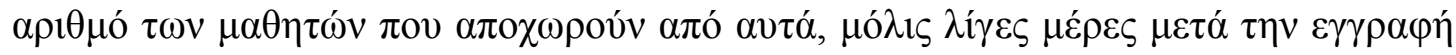

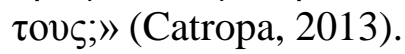

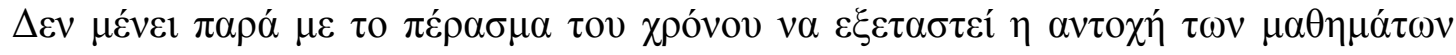

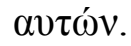




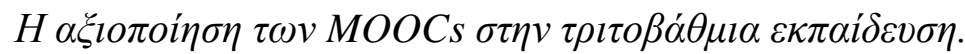

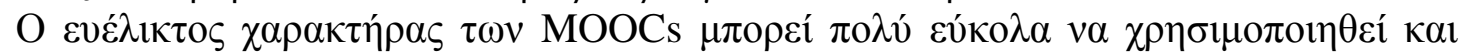

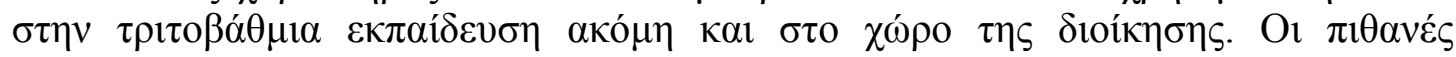

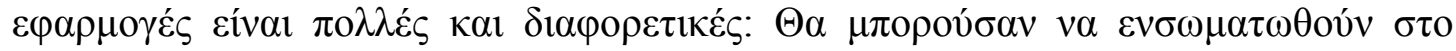

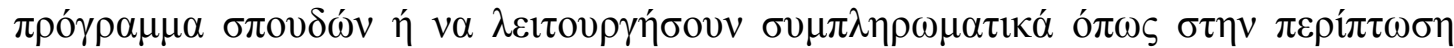
$\tau \omega v$ Khean Academy (https://www.khanacademy.org), Udacity (www.udacity.com)



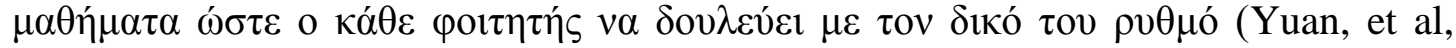
2013).

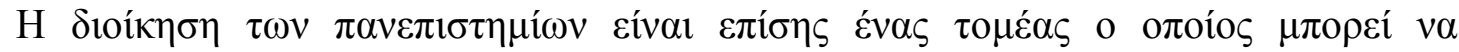

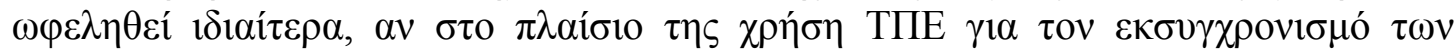

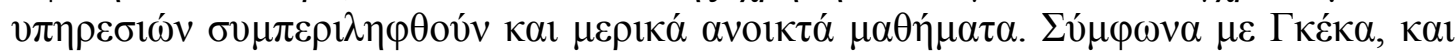

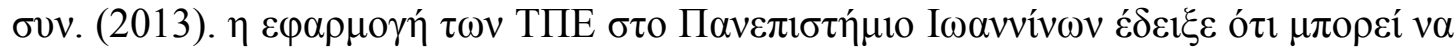

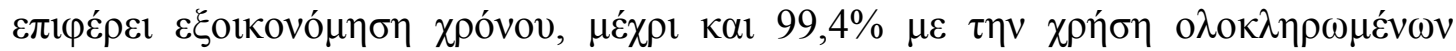

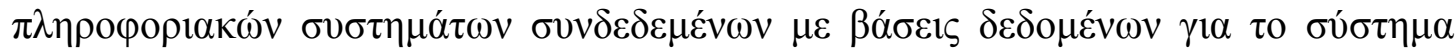

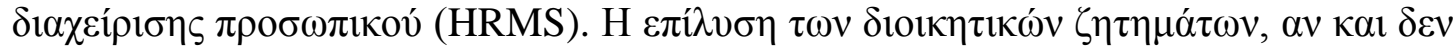

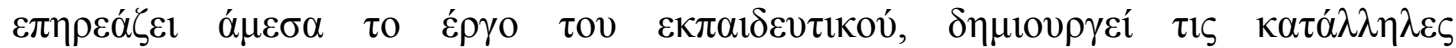

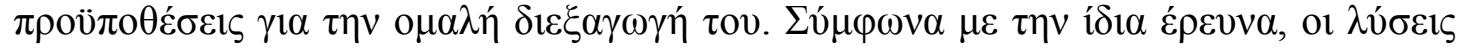

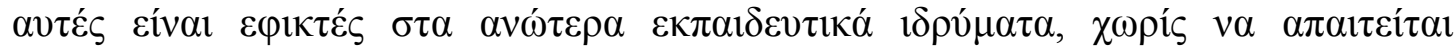

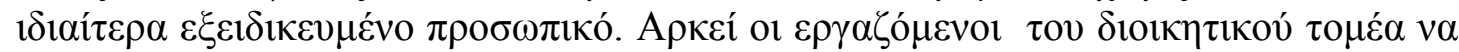

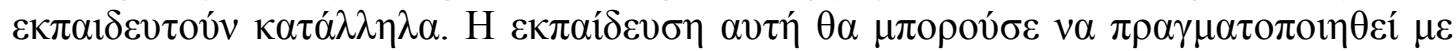

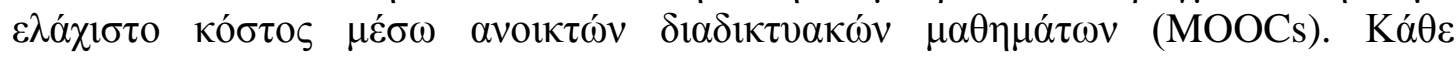

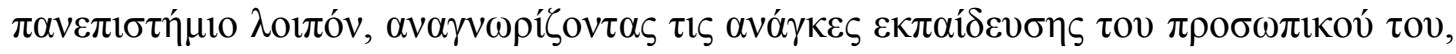

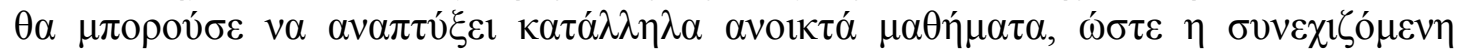

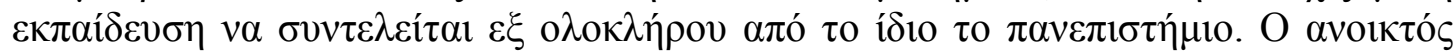

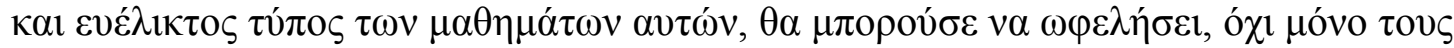

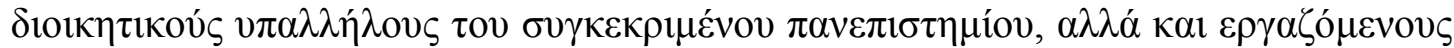

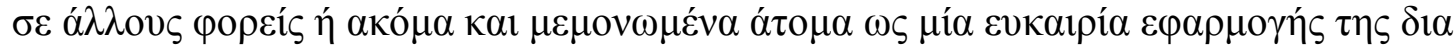
Bíov $\mu \alpha \dot{\theta} \theta \eta \sigma \eta \varsigma$.

\section{$\Sigma v \mu \pi \varepsilon \rho \alpha ́ \sigma \mu \alpha \tau \alpha$}

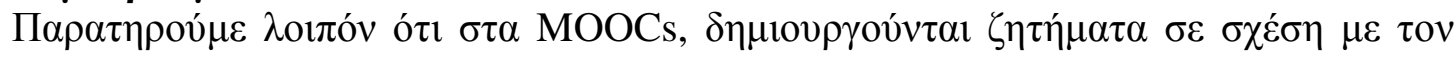

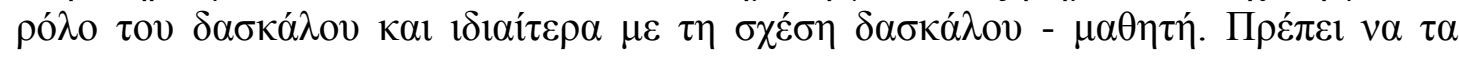

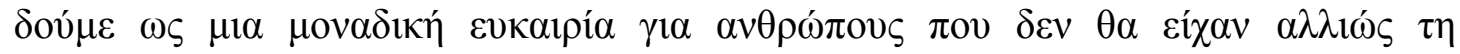

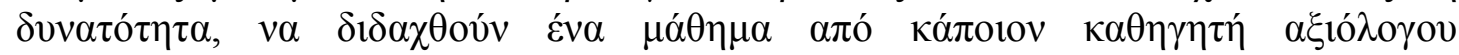

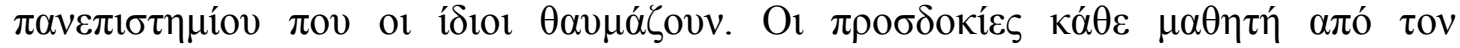



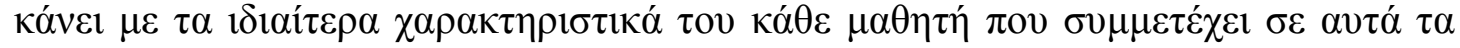

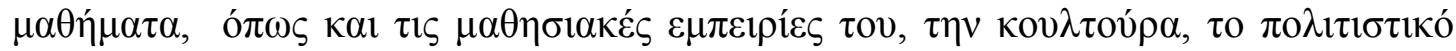

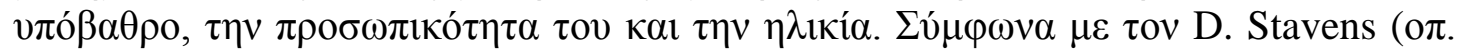

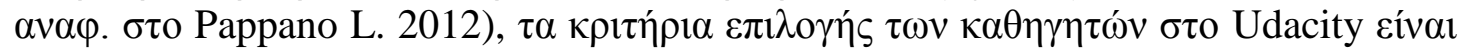

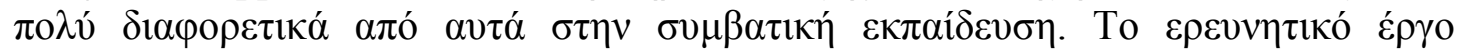

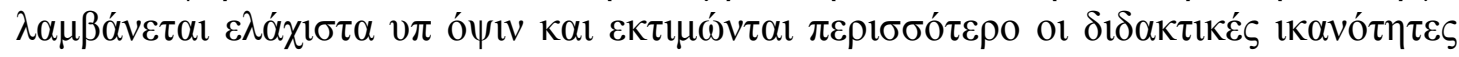



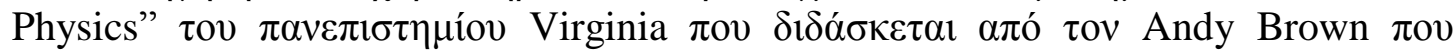

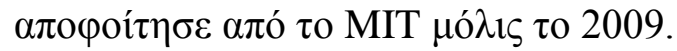

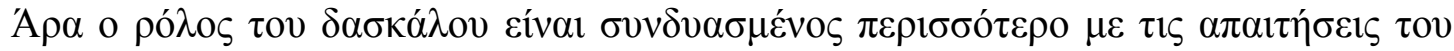

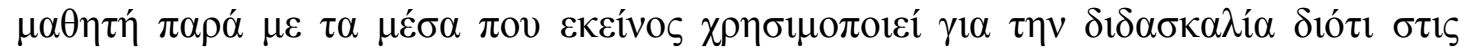

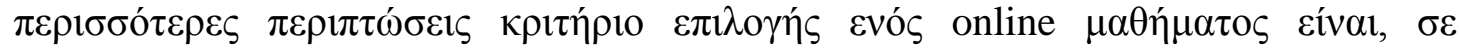






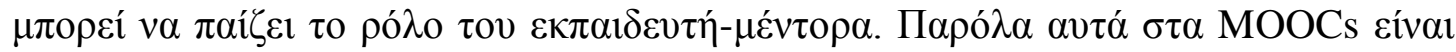

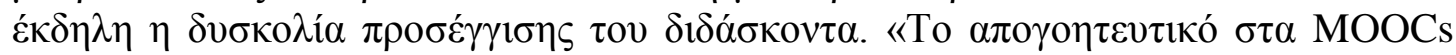
eíval ó

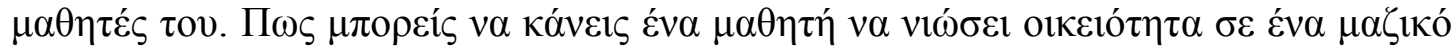

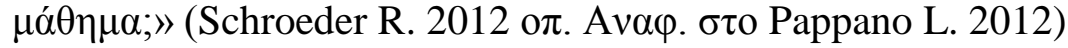

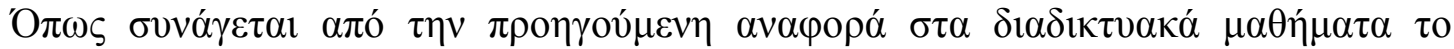



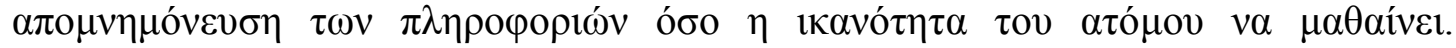

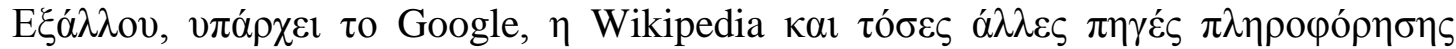

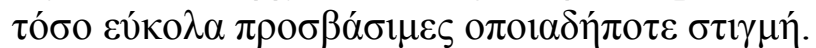

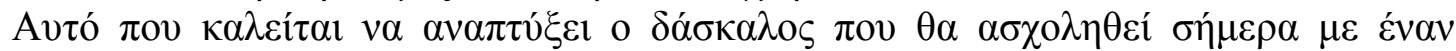

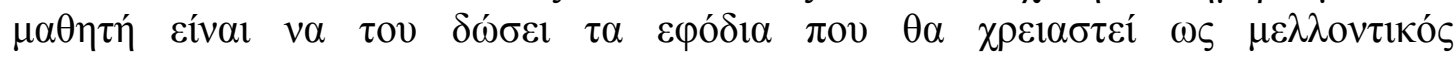

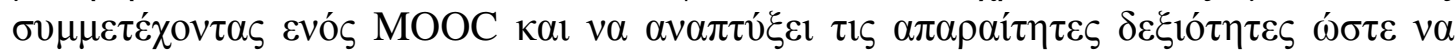

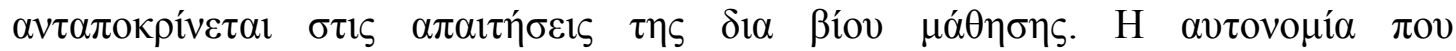

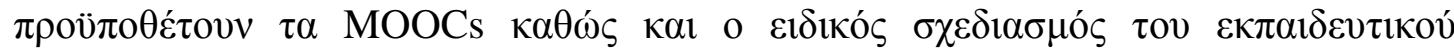

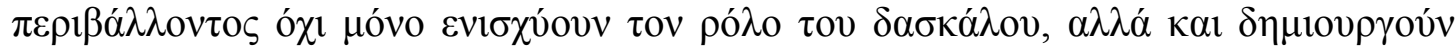

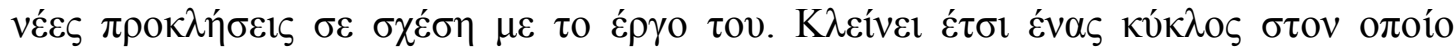

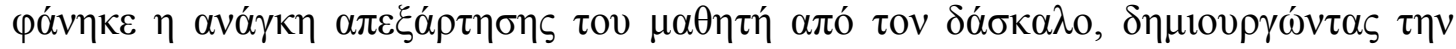



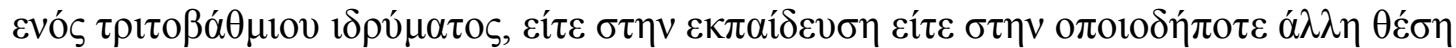

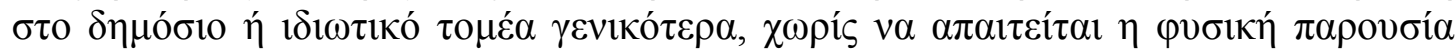
$\tau$ cov $\delta \alpha \sigma \kappa \alpha ́ \lambda o v$.

\section{Avapopés}

Catropa, Dayna (February 24, 2013). "Big (MOOC) Data". Inside Higher Ed. Retrieved July 25, 2013.

Coolahan John (2002). Teacher Education and the Teaching Career in an Era of Lifelong Learning, OECD Publishing

Day Christopher (2000). Teachers in the Twenty-first Century: Time to renew the vision, Teachers and Teaching: Theory and Practice Volume 6, Issue 1, 2000

Degree of Freedom - an adventure in online learning, MOOC Components - Assessment, March 22 , 2013

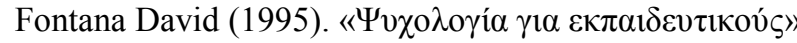

Jacobs A. J. Two cheers for wed U (2013) Retrieved 15-09-2013 http://www.nytimes.com/2013/04/21/opinion/sunday/grading-the-moocuniversity.html?pagewanted $=$ all\& $\mathrm{r}=0$

Keegan Desmond (2000). "Foundations of distance education"

Knowledge@ Wharton "MOOCs on the Move: How Coursera Is Disrupting the Traditional Classroom" (text and video).. University of Pennsylvania. November 7, 2012. Retrieved June 29, 2013.

KourtopoulosA., Gallagher M.S., et al, “ Emotive vocabulary In MOOCs: Context and participant retention" http://www.eurodl.org/?article=507 Retrieved June 29, 2013

MacKay, R.F. (April 11, 2013). "Learning analytics at Stanford takes huge leap forward with MOOCs". Stanford Report. Stanford University. Retrieved June 22, 2013.

Moore Michael G., Kearsley Greg (2012). Distance Education: A Systems View of Online Learning, 3rd ed.

Race Phil (1993). "The Open Learning Handbook"

Siemens, George. "MOOC or Mega-Connectivism Course". Retrieved 2009-01-28. http://ltc.umanitoba.ca/connectivism/

Siemens, George; Stephen Downes. "Connectivism and Connective Knowledge". Retrieved 2009-0128. http://ltc.umanitoba.ca/connectivism/

Searby, Mike, and Tim Ewers (1997) "An evaluation of the use of peer assessment in higher education: A case study in the School of Music" p.371

Villegas, A. M., \& Lucas, T. (2002). Educating culturally responsive teachers: A coherent approach. Albany, NY: SUNY Press 
Yuan, Li, and Stephen Powell. MOOCs and Open Education: Implications for Higher Education White Paper. University of Bolton: CETIS, 2013. pp. 7-8.

Young, Jeffrey R. (January 27, 2013). "The Object Formerly Known as the Textbook". Chronicle of Higher Education. Retrieved March 14, 2013.

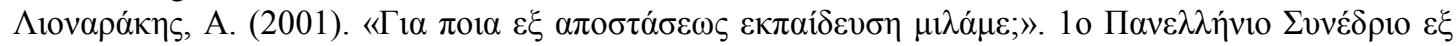

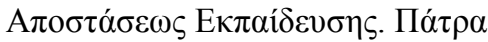

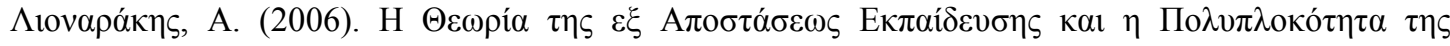

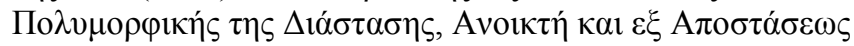

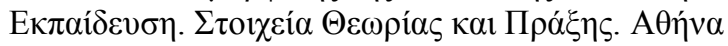

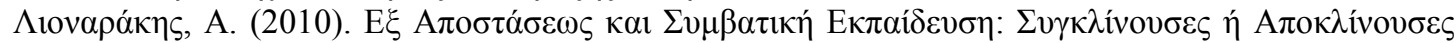

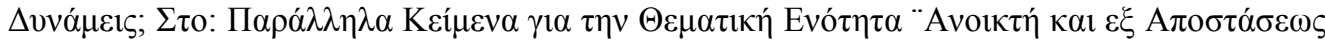

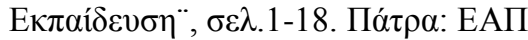

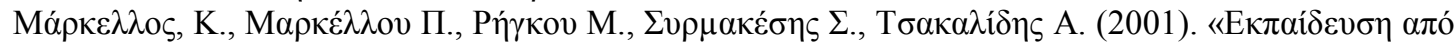



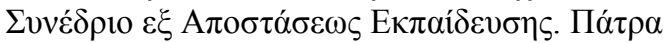

Pappano L. (2012). The year of MOOC. Retrieved 15-09-2013 http://www.nytimes.com/2012/11/04/education/edlife/massive-open-online-courses-aremultiplying-at-a-rapid-pace.html?pagewanted=all\&_r=0

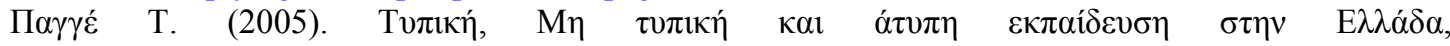
http://equipe.up.pt/RESOURCES/Casestudies/original_language/ioannina_GR.doc

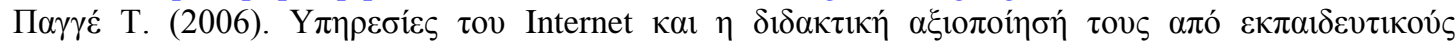

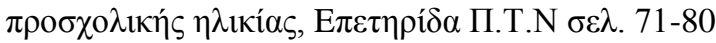

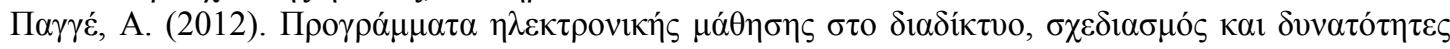

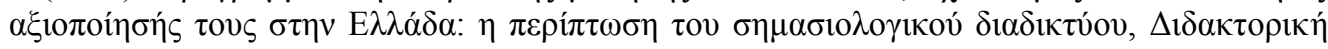

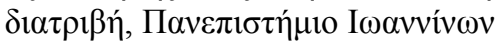

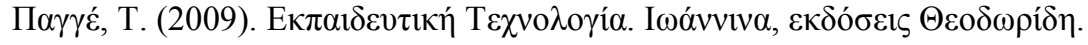

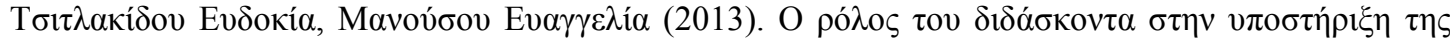

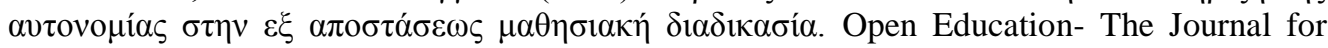
Open and Distance Education and Educational Technology. Vol.9, $\mathrm{N}^{\mathrm{o}} 1,2013$ 